Supporting information for:

\title{
Immobilization Strategies for Enhancing Sensitivity of Electrochemical Aptamer-Based Sensors
}

\author{
Yingzhu Liu†, Juan Canoura†, Obtin Alkhamis and Yi Xiao* \\ Department of Chemistry and Biochemistry, Florida International University, 11200 SW 8th \\ Street, Miami, FL, 33199. \\ $\dagger$ These authors contributed equally to this work. \\ *Corresponding author: yxiao2@fiu.edu
}




\section{Table of Contents}

Supporting Table

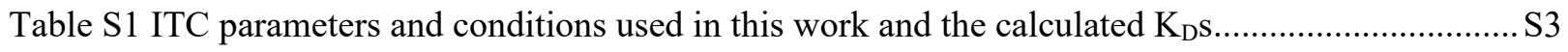

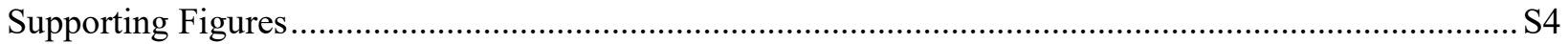

Figure S1 ITC characterization of the affinity of ADE-25 for adenosine............................................... S4

Figure S2 Adenosine detection with electrodes prepared using traditional approach ........................... S5

Figure S3 Simulated binding curve for ADE-25 based on its affinity for adenosine in high-salt PBS .... S6

Figure S4 Surface coverage of electrodes fabricated with ADE-25-MB via different methods............... S6

Figure S5 Sensor performance of electrodes fabricated with COC-32-MB via different methods .......... S7

Figure S6 ITC characterization of the affinity of COC-32 for cocaine …............................................ S8

Figure S7 Simulated binding curve for COC-32 based on its affinity for cocaine in high-salt PBS ........ S8

Figure S8 Surface coverage of electrodes modified with COC-32-MB and cocaine in high-salt PBS .... S9

Figure S9 Sensor performance of electrodes fabricated with COC-32-MB alone in different buffers..... S9

Figure S10 Simulated binding curve for COC-32 based on the affinity for cocaine in low-salt PBS .... S10

Figure S11 Surface coverage of electrodes modified with COC-32-MB with different methods .......... S10

Figure S12 Performance of electrodes fabricated with COC-32-MB and target in different buffers ..... S1 1

Figure S13 Sensitivity and LOD for cocaine detection in 50\% saliva with different electrodes............ S11

Figure S14 Simulated binding curve for SC-34 based on affinity for MDPV in low-salt Tris buffer... S12

Figure S15 SWV spectra of electrodes fabricated using SC-34-MB and MDPV ............................... S12

Figure S16 Detection of MDPV in 50\% urine using electrodes fabricated via different methods ......... S13

Figure S17 ITC characterization of the affinity of SC-34 for MDPV ….............................................. 13

Figure S18 Simulated binding curve for SC-34 based on affinity for MDPV in high-salt PBS............ S14

Figure S19 Performance of electrodes fabricated with SC-34-MB and MDPV in different buffers ...... S14

Figure S20 Performance of electrodes fabricated with SC-34-MB via different methods ................... S15

Figure S21 ITC characterization of SC-34 affinity for MDPV in high-salt Tris buffer .......................S16

Figure S22 Surface coverage of electrodes fabricated with SC-34-MB in various $\mathrm{pH}$ buffers ............. S16

Figure S23 Performance of electrodes fabricated with SC-34-MB via different methods .................... S17

Figure S24 ITC characterization of the $\mathrm{pH}$-dependence of SC-34 affinity for MDPV .......................S18 
Table S1. ITC parameters and conditions used in this work and the calculated $\mathrm{K}_{\mathrm{DS}} \mathrm{pH}$ of the buffer is 7.4 unless stated otherwise.

\begin{tabular}{|c|c|c|c|}
\hline Cell content & Syringe content & Buffer & $K_{D}(\mu M)$ \\
\hline $20 \mu \mathrm{M} \mathrm{SC}-34$ & $400 \mu \mathrm{M}(-)-\mathrm{MDPV}$ & Low-salt Tris & $0.33 \pm 0.04$ \\
\hline $20 \mu \mathrm{M} \mathrm{SC}-34$ & $400 \mu \mathrm{M}(-)-\mathrm{MDPV}$ & Low-salt PBS & $0.38 \pm 0.03$ \\
\hline $20 \mu \mathrm{M} \mathrm{SC}-34$ & $350 \mu \mathrm{M}(-)-\mathrm{MDPV}$ & Low-salt PBS (pH 6.0) & $0.23 \pm 0.02$ \\
\hline $20 \mu \mathrm{M} \mathrm{SC}-34$ & $350 \mu \mathrm{M}(-)-\mathrm{MDPV}$ & Low-salt PBS (pH 7.0) & $0.38 \pm 0.02$ \\
\hline $20 \mu \mathrm{M} \mathrm{SC}-34$ & $350 \mu \mathrm{M}(-)-\mathrm{MDPV}$ & Low-salt PBS (pH 7.4) & $0.56 \pm 0.03$ \\
\hline $20 \mu \mathrm{M} \mathrm{SC}-34$ & $350 \mu \mathrm{M}(-)-\mathrm{MDPV}$ & Low-salt PBS (pH 8.0) & $0.69 \pm 0.03$ \\
\hline $20 \mu \mathrm{M} \mathrm{SC}-34$ & $300 \mu \mathrm{M}(-)-\mathrm{MDPV}$ & High-salt Tris & $1.80 \pm 0.05$ \\
\hline $20 \mu \mathrm{M} \mathrm{SC}-34$ & $\begin{array}{l}200 \mu \mathrm{M}(-)-\mathrm{MDPV} \\
(2 \times, \text { back-to-back })\end{array}$ & High-salt PBS & $1.50 \pm 0.04$ \\
\hline $20 \mu \mathrm{M} \mathrm{COC}-32$ & $800 \mu \mathrm{M}$ cocaine & Low-salt Tris & $5.8 \pm 0.3$ \\
\hline $20 \mu \mathrm{M}$ COC-32 & $800 \mu \mathrm{M}$ cocaine & Low-salt PBS & $5.9 \pm 0.3$ \\
\hline $80 \mu \mathrm{M} \mathrm{COC}-32$ & $4000 \mu \mathrm{M}$ cocaine & High-salt PBS & $70.4 \pm 0.8$ \\
\hline $20 \mu \mathrm{M} \mathrm{ADE}-25$ & $\begin{array}{l}1200 \mu \mathrm{M} \text { adenosine } \\
(2 \times, \text { back-to-back })\end{array}$ & Low-salt Tris & $\begin{array}{l}\mathrm{K}_{\mathrm{D} 1}=7.4 \pm 0.1 \\
\mathrm{~K}_{\mathrm{D} 2}=103 \pm 0.5 \\
\mathrm{~K}_{1 / 2}=27.6 \pm 0.2\end{array}$ \\
\hline $20 \mu \mathrm{M}$ ADE-25 & $2500 \mu \mathrm{M}$ Adenosine & High-salt PBS & $\begin{array}{l}\mathrm{K}_{\mathrm{D} 1}=36.7 \pm 2.4 \\
\mathrm{~K}_{\mathrm{D} 2}=14.5 \pm 0.6 \\
\mathrm{~K}_{1 / 2}=23.1 \pm 0.8\end{array}$ \\
\hline
\end{tabular}



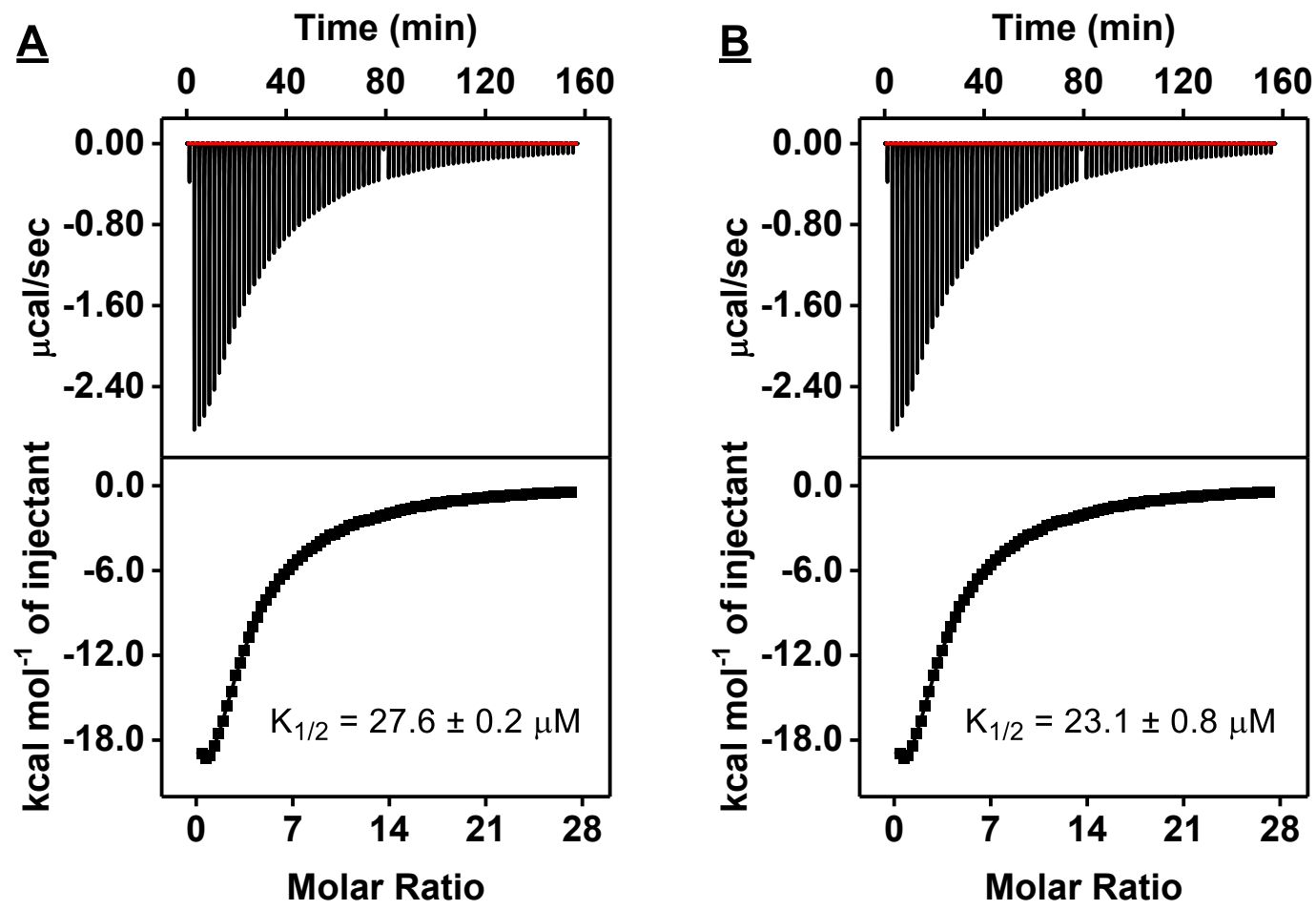

Figure S1. Characterization of the affinity of ADE-25 for adenosine using isothermal titration calorimetry (ITC). Top panels present raw data showing the heat generated from each titration of adenosine to ADE-25 in (A) low-salt Tris buffer (10 mM Tris-HCl, $20 \mathrm{mM} \mathrm{NaCl}, 0.5 \mathrm{mM} \mathrm{MgCl}$, $\mathrm{pH}$ 7.4) or (B) high-salt PBS (1.6 mM NaH $2 \mathrm{PO}_{4}, 8.4 \mathrm{mM} \mathrm{Na}_{2} \mathrm{HPO}_{4}, 1 \mathrm{M} \mathrm{NaCl}, 1 \mathrm{mM} \mathrm{MgCl}, \mathrm{pH}$ 7.2), while bottom panels show the integrated heat of each titration after correcting for dilution heat of the titrant. ITC data were fitted with a two-site sequential binding model. $\mathrm{K}_{1 / 2}=\left(\mathrm{K}_{\mathrm{D} 1} \times\right.$ $\left.\mathrm{K}_{\mathrm{D} 2}\right)^{1 / 2}$ 

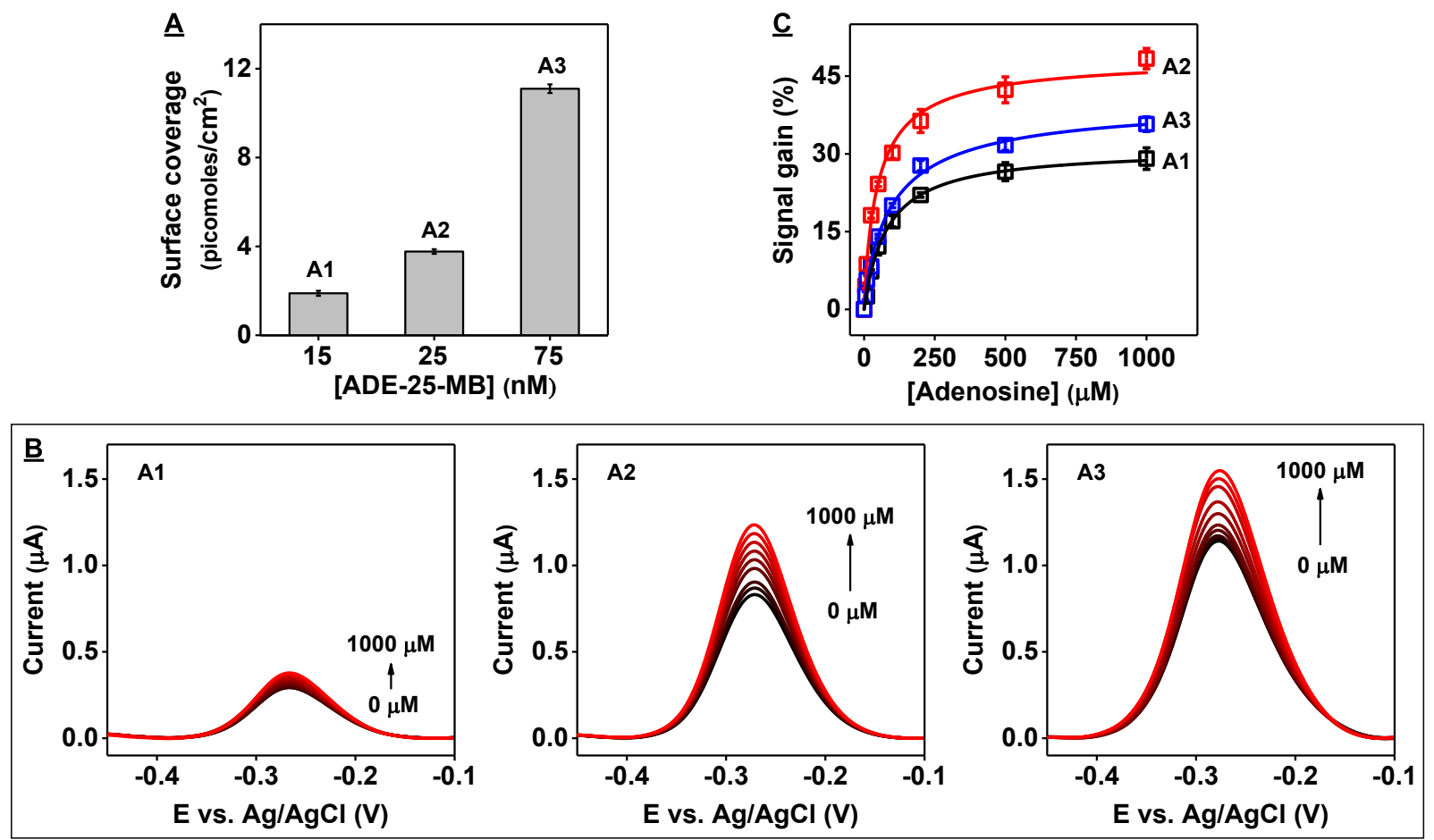

Figure S2. Adenosine detection with electrodes modified with ADE-25-MB using the conventional immobilization approach. (A) Aptamer surface coverage of electrodes fabricated using $15 \mathrm{nM}$ (A1), $25 \mathrm{nM}$ (A2), or $75 \mathrm{nM}$ (A3) ADE-25-MB in the absence of target. (B) Square wave voltammograms (SWV) and (C) calibration curves for adenosine detection produced by the different $\mathrm{E}-\mathrm{AB}$ sensors with $0-1,000 \mu \mathrm{M}$ adenosine. Error bars represent the standard deviation of measurements from three independently fabricated electrodes. 


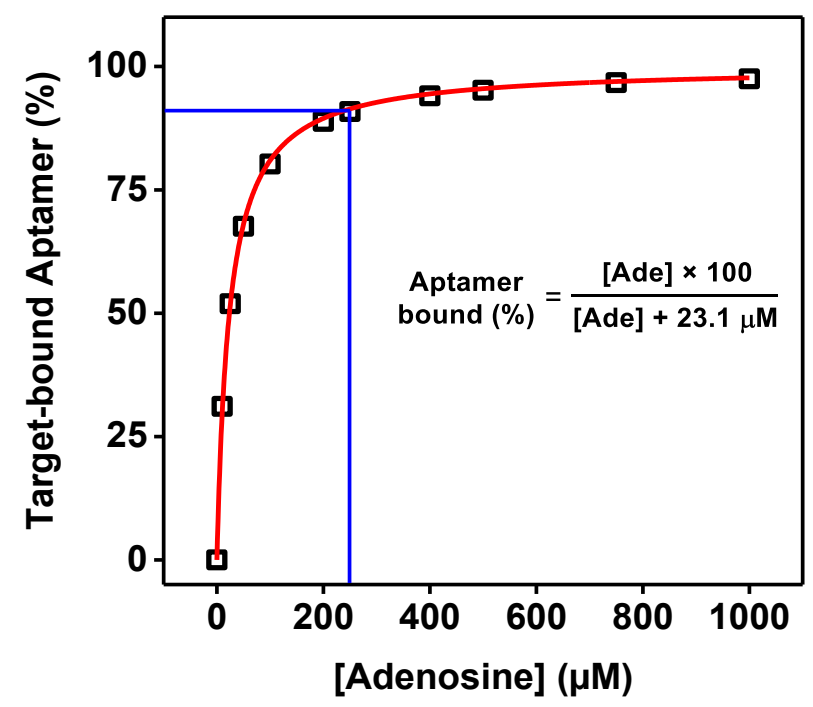

Figure S3. Simulated binding curve for ADE-25 based on its affinity for adenosine in high-salt PBS $\left(\mathrm{K}_{1 / 2}=23.1 \mu \mathrm{M}\right)$. Blue lines indicate that $\sim 91 \%$ of the aptamer is bound in the presence of $250 \mu \mathrm{M}$ adenosine.

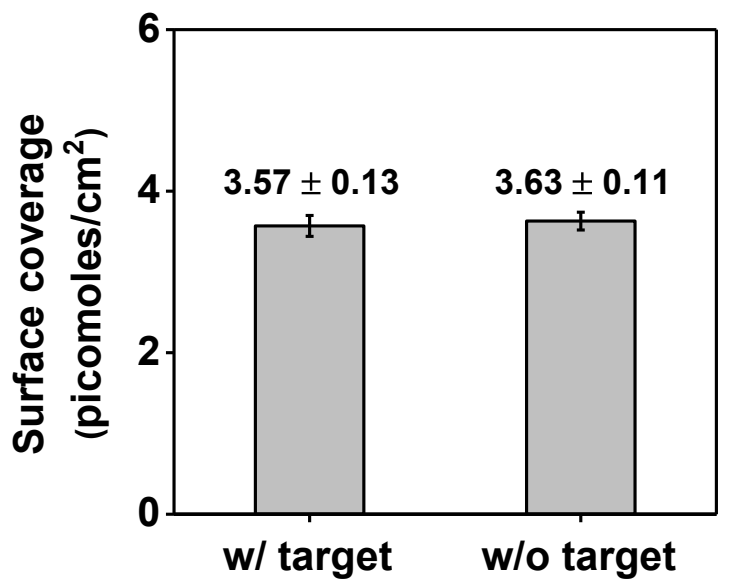

Figure S4. Aptamer surface coverages of electrodes fabricated by immobilizing the aptamer in the presence or absence of $250 \mu \mathrm{M}$ adenosine. Error bars represent the standard deviation of measurements from three independently fabricated electrodes. 

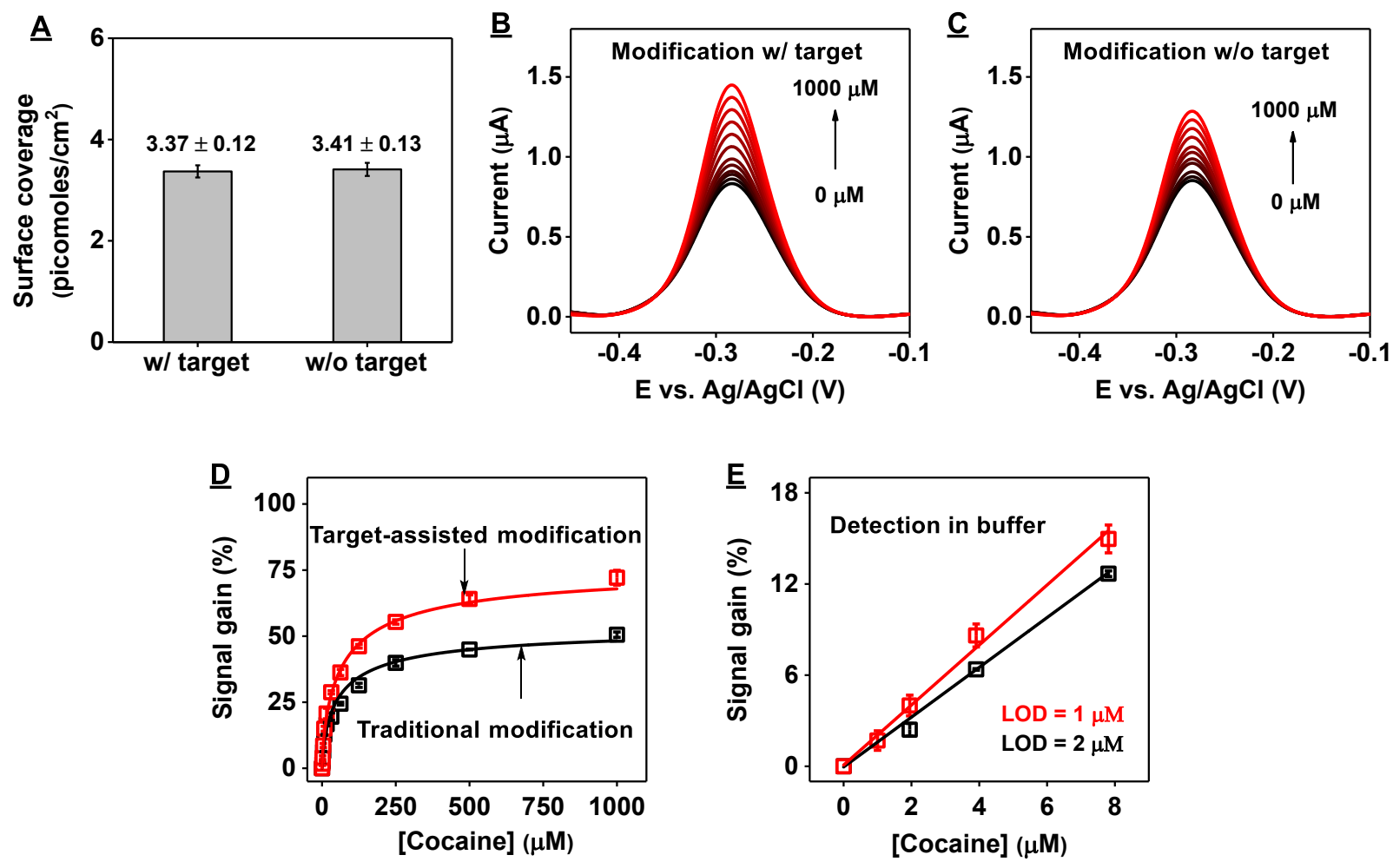

Figure S5. Performance of electrodes fabricated with COC-32-MB via target-assisted immobilization or conventional means in high-salt PBS. (A) Surface coverage of electrodes fabricated by immobilizing the aptamer in the presence (w/ target) or absence (w/o target) of 250 $\mu \mathrm{M}$ cocaine. SWV from electrodes fabricated via (B) target-assisted immobilization or (C) traditional means (aptamer only) and challenged with $0-1000 \mu \mathrm{M}$ cocaine. (D) Calibration curves for detection of cocaine derived from the spectra shown in $\mathbf{B}$ (red) and $\mathbf{C}$ (black). (E) Sensitivity and LOD of electrodes fabricated using target-assisted immobilization (red) or traditional means (black). Error bars represent the standard deviation of measurements from three independently fabricated electrodes. 

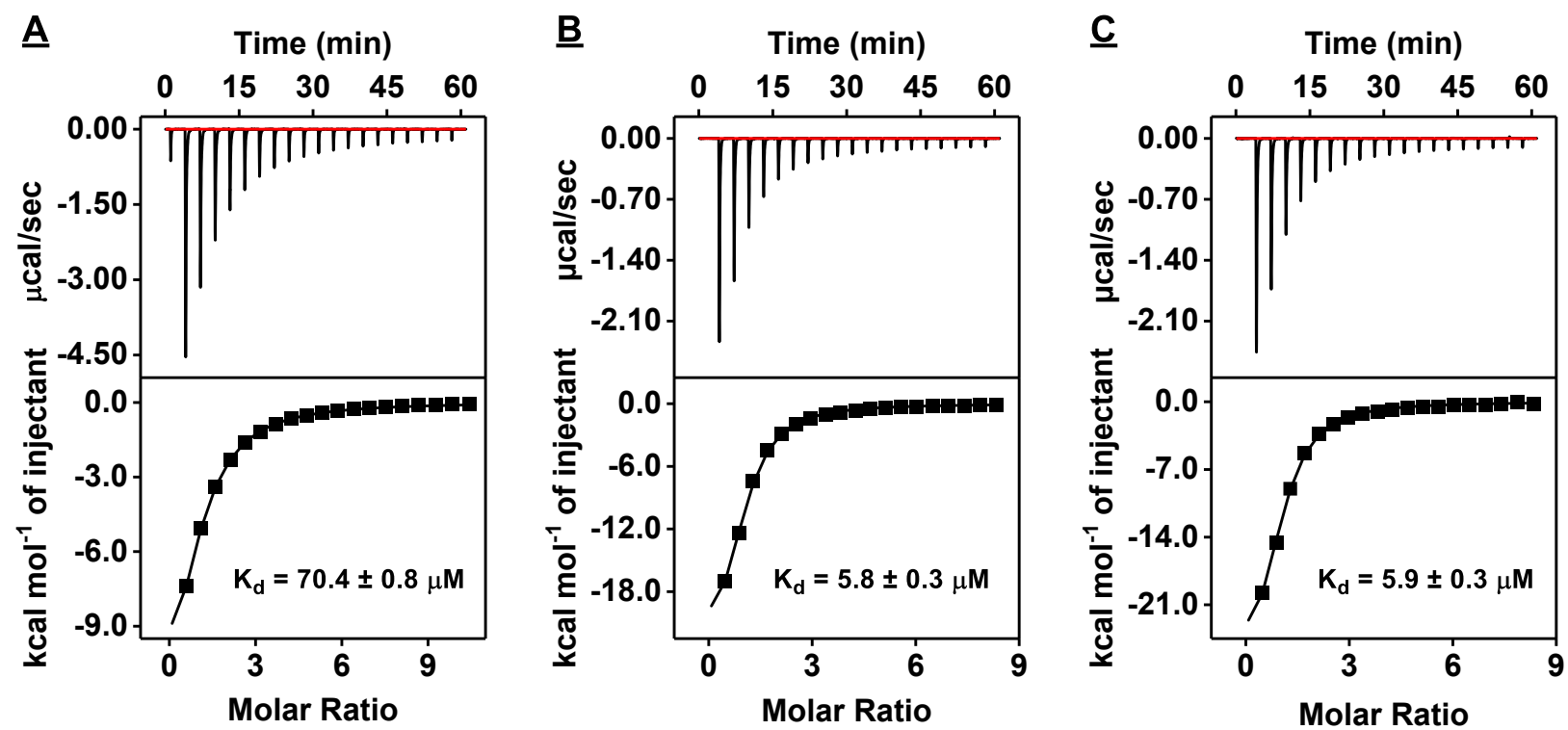

Figure S6. Characterization of the affinity of COC-32 for cocaine using ITC. Top panels present raw data showing the heat generated from each titration of cocaine to COC-32 in (A) high-salt PBS, (B) low-salt PBS, or (C) low-salt Tris buffer, while bottom panels show the integrated heat of each titration after correcting for dilution heat of the titrant. ITC data were fitted with a singlesite binding model.

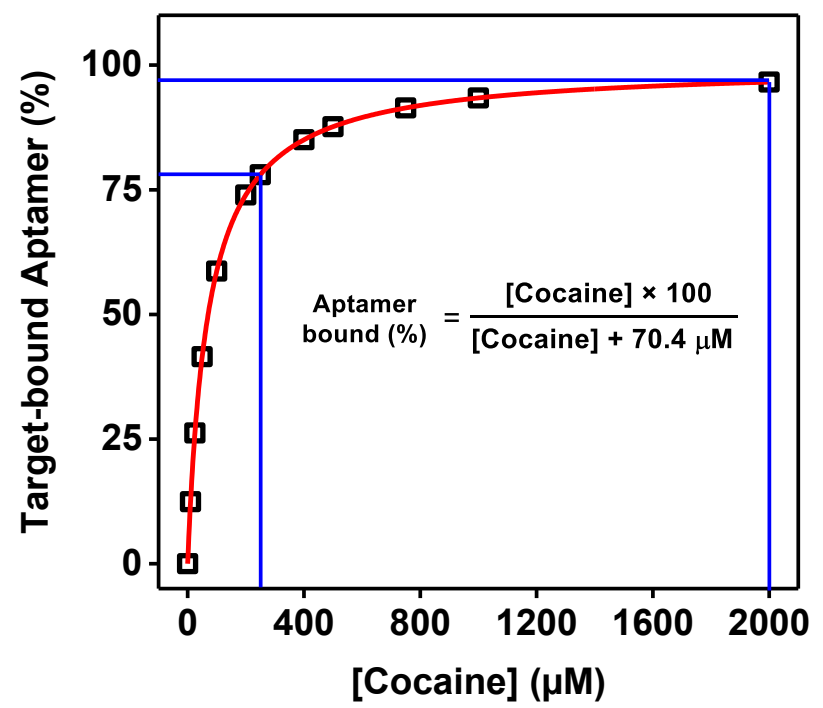

Figure S7. Simulated binding curve for COC-32 based on its affinity for cocaine in high-salt PBS $\left(\mathrm{K}_{\mathrm{D}}=70.4 \mu \mathrm{M}\right)$. The blue lines indicate that $\sim 78 \%$ and $96 \%$ of the aptamer is bound in the presence of $250 \mu \mathrm{M}$ and $2 \mathrm{mM}$ cocaine, respectively. 


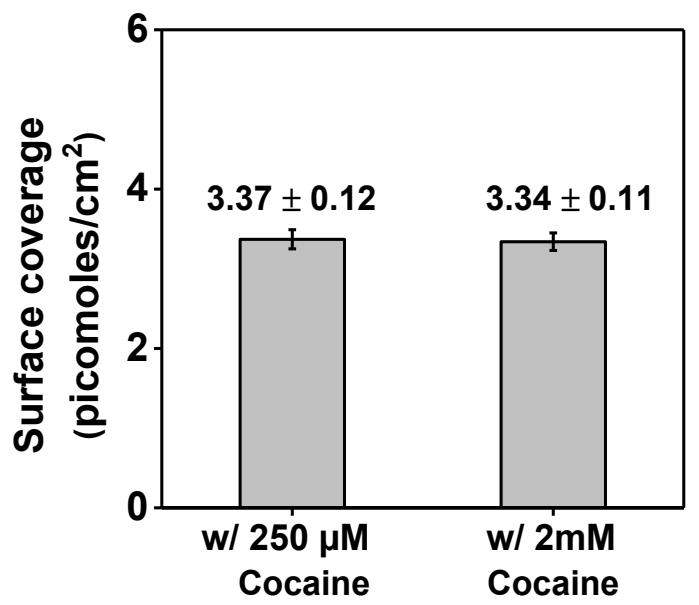

Figure S8. Surface coverage of electrodes modified with COC-32-MB via target-assisted immobilization in high-salt PBS in the presence of $250 \mu \mathrm{M}$ or $2 \mathrm{mM}$ cocaine. Error bars represent the standard deviation of measurements from three different electrodes.
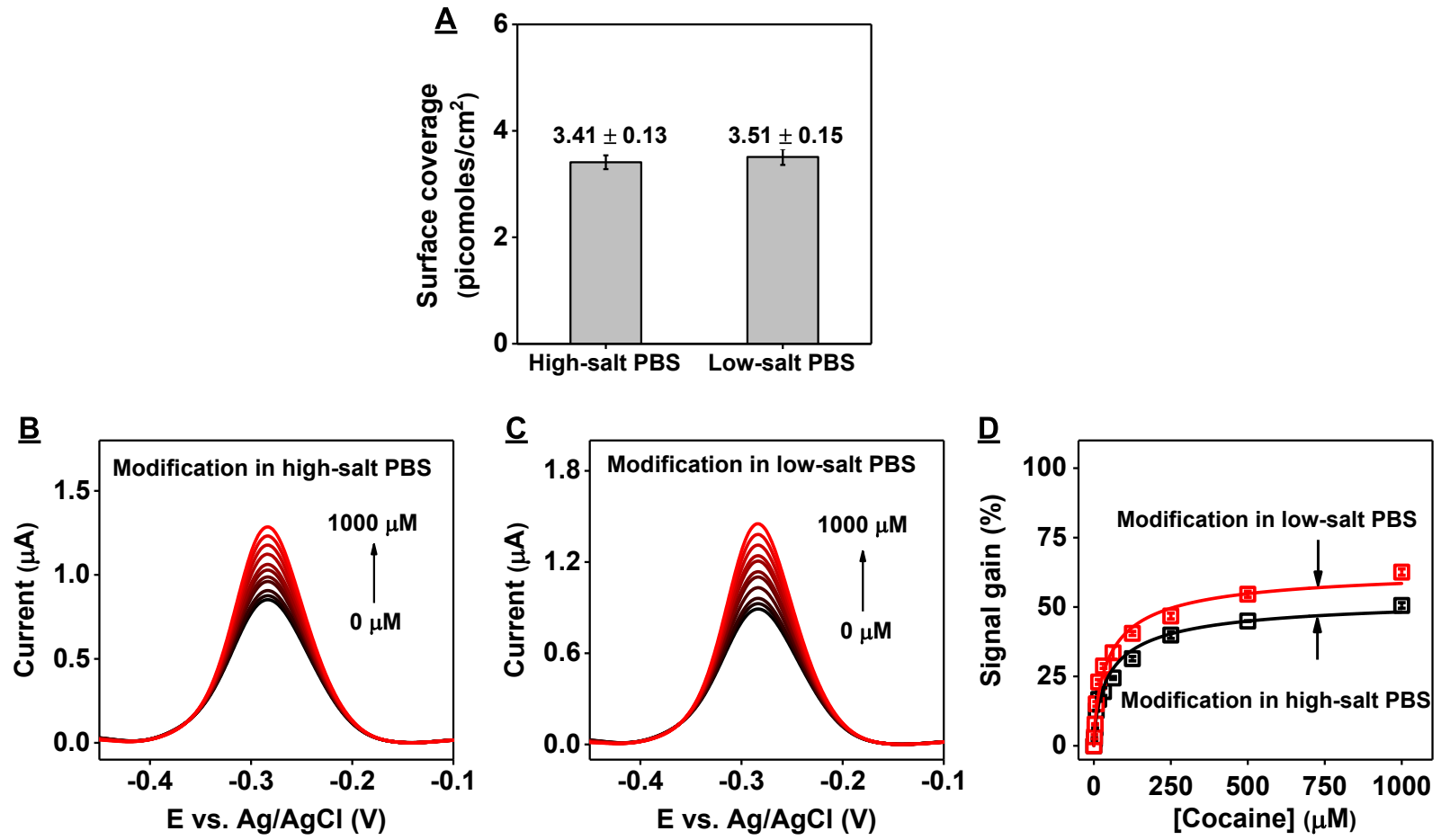

Figure S9. Performance of electrodes fabricated with COC-32-MB via conventional means in different buffers. (A) Surface coverage of electrodes fabricated by immobilizing the aptamer in high-salt or low-salt PBS. SWV produced by challenging electrodes fabricated in (B) high-salt or (C) low-salt PBS with 0-1000 $\mu \mathrm{M}$ cocaine. (D) Calibration curves for detection of cocaine derived from the spectra shown in $\mathbf{B}$ (black) and $\mathbf{C}$ (red). Error bars represent the standard deviation of measurements from three independently fabricated electrodes. 


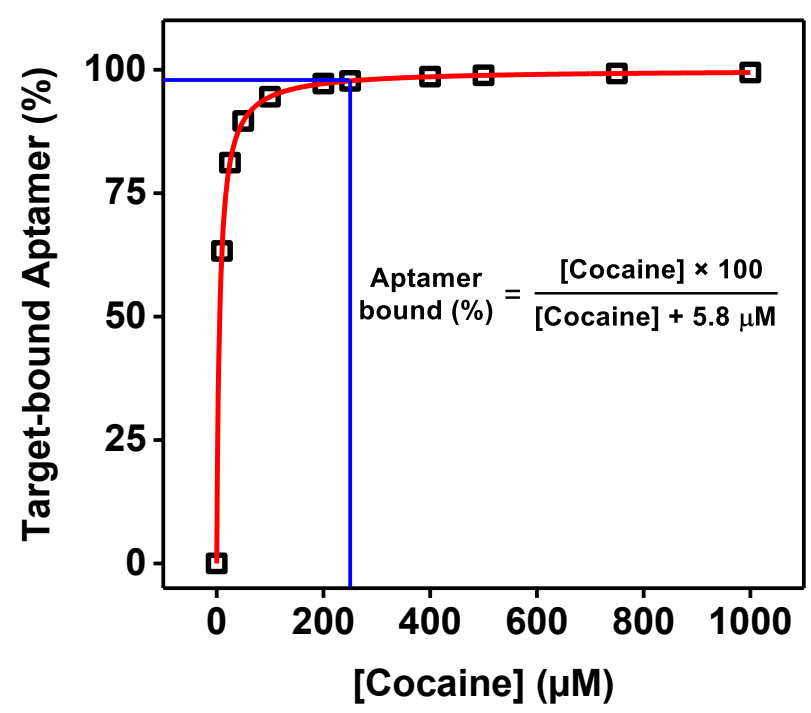

Figure S10. Simulated binding curve for COC-32 based on the affinity for cocaine in low-salt PBS $\left(\mathrm{K}_{\mathrm{D}}=5.8 \mu \mathrm{M}\right)$. The blue lines indicate that $96 \%$ of the aptamer is bound in the presence of $250 \mu \mathrm{M}$ cocaine.

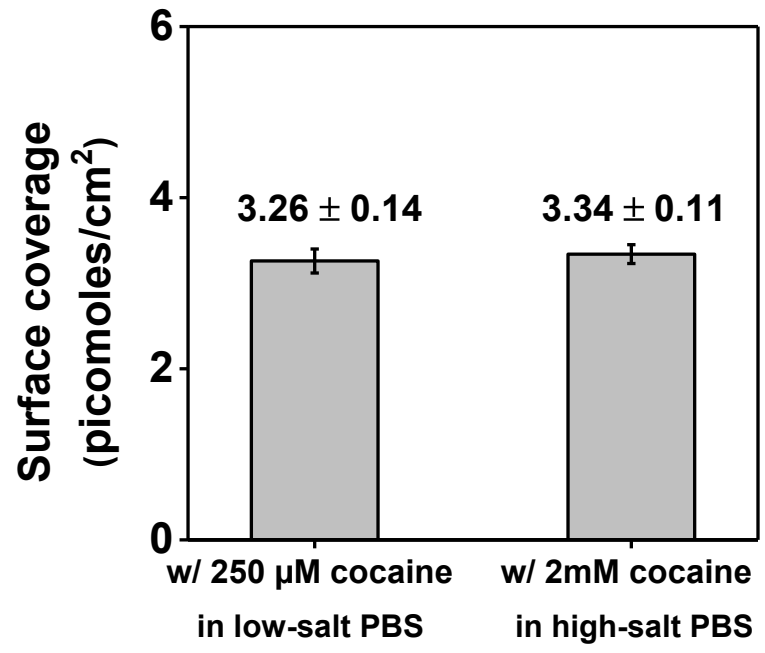

Figure S11. Surface coverage of electrodes modified with COC-32-MB via target-assisted immobilization in low-salt PBS with $250 \mu \mathrm{M}$ or in high-salt PBS with $2 \mathrm{mM}$ cocaine. Error bars represent the standard deviation of measurements from three different electrodes. 

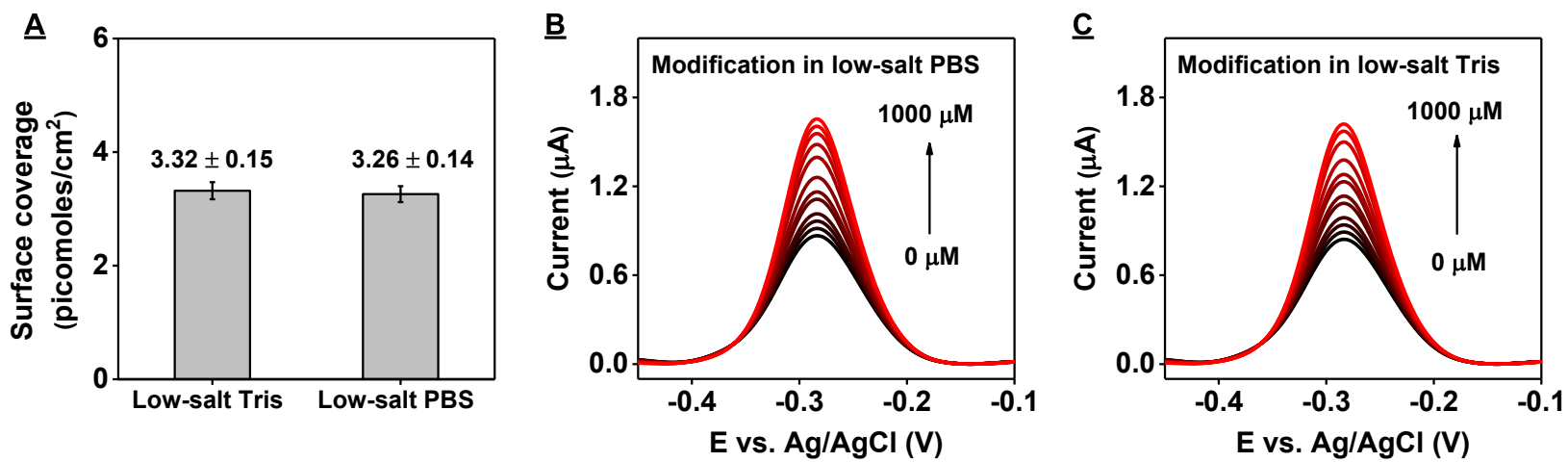

Figure S12. Performance of electrodes fabricated with COC-32-MB via target-assisted immobilization in different buffers. (A) Aptamer surface coverages of electrodes fabricated by immobilizing the aptamer in low-salt Tris buffer or low-salt PBS with $250 \mu \mathrm{M}$ cocaine. SWV produced by challenging the electrodes fabricated in low-salt (B) PBS or (C) Tris buffer with 0 $1000 \mu \mathrm{M}$ cocaine. Error bars represent the standard deviation of measurements from three independently fabricated electrodes.

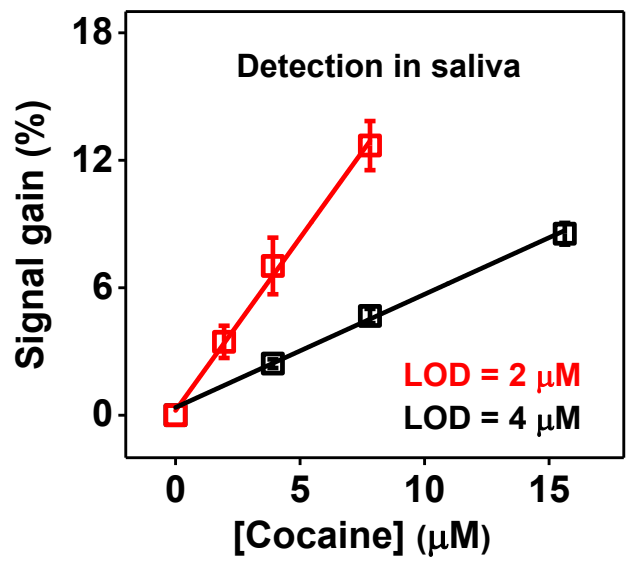

Figure S13. Sensitivity and LOD for cocaine detection in 50\% saliva with electrodes fabricated using COC-32-MB via target-assisted immobilization in low-salt Tris buffer with $250 \mu \mathrm{M}$ cocaine (red) or conventional means in high-salt PBS (black). Error bars represent the standard deviation of measurements from three independently fabricated electrodes. 


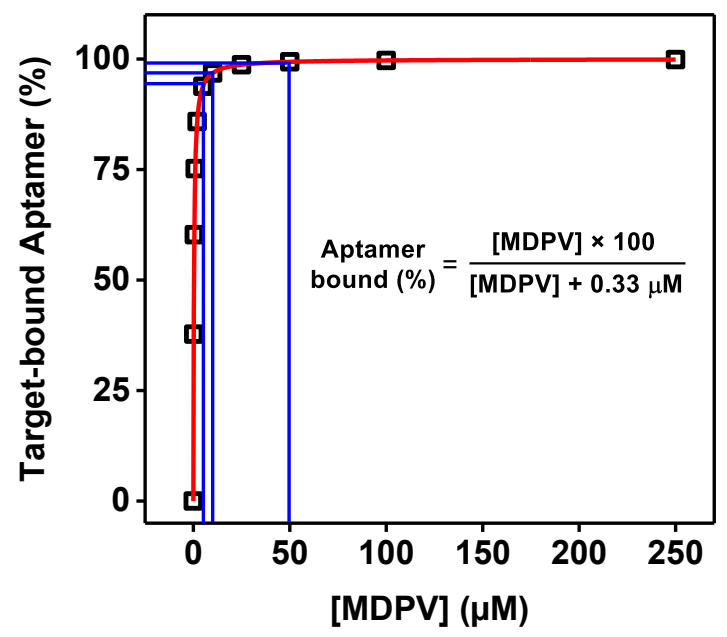

Figure S14. Simulated binding curve for SC-34 based on affinity for MDPV in low-salt Tris buffer $\left(\mathrm{K}_{\mathrm{D}}=0.33 \mu \mathrm{M}\right)$. The blue lines indicate that $94 \%, 97 \%$, and $99 \%$ of the aptamer is bound in the presence of 5,10 , and $50 \mu \mathrm{M}$ MDPV, respectively.
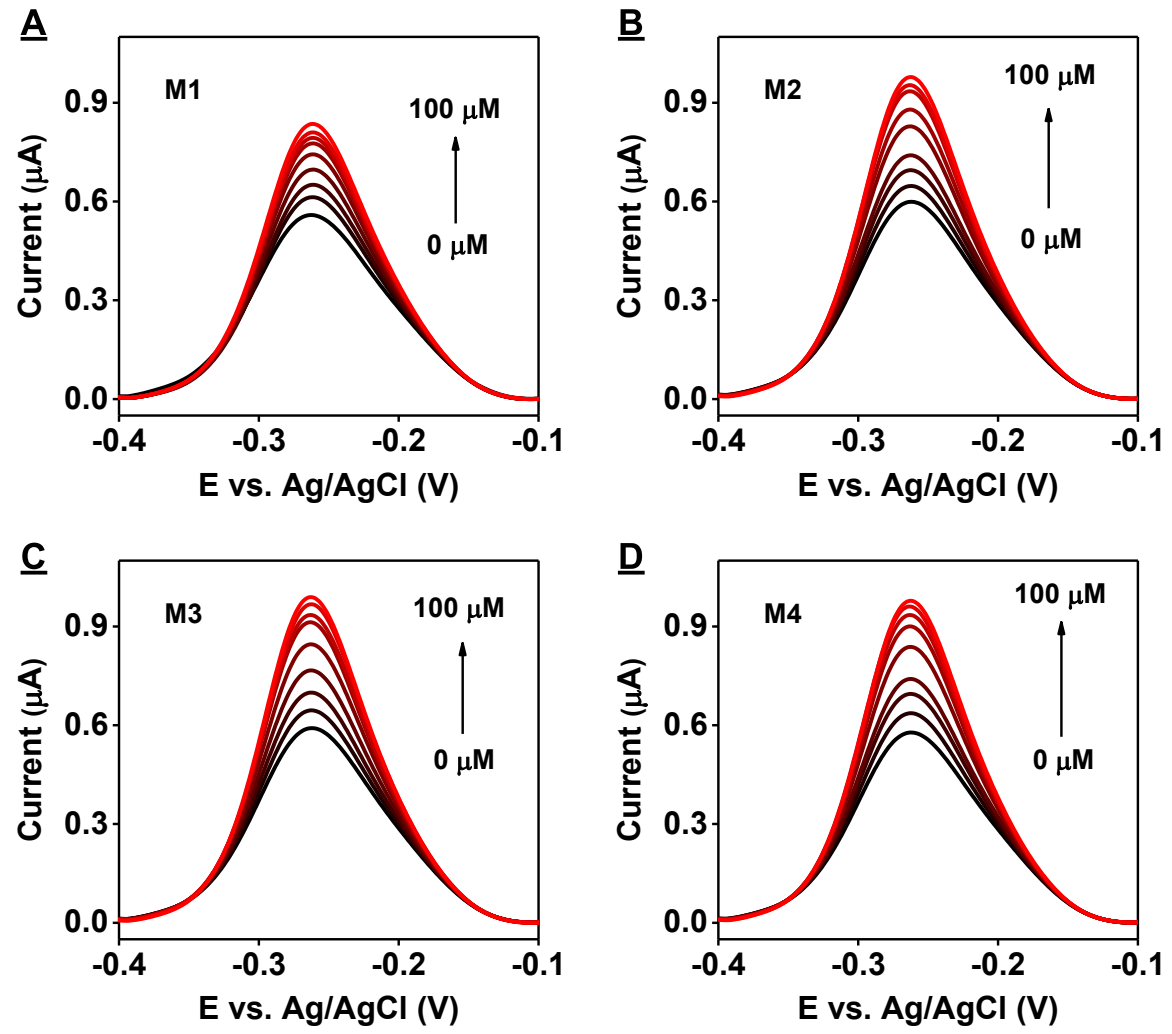

Figure S15. Electrodes fabricated using SC-34-MB via target-assisted aptamer immobilization with various concentrations of MDPV. SWV for electrodes modified with SC-34-MB in the presence of (A) $0 \mu \mathrm{M}$, (B) $5 \mu \mathrm{M}$, (C) $10 \mu \mathrm{M}$, and (D) $50 \mu \mathrm{M}$ MDPV after challenging with 0-100 $\mu \mathrm{M}$ MDPV. 

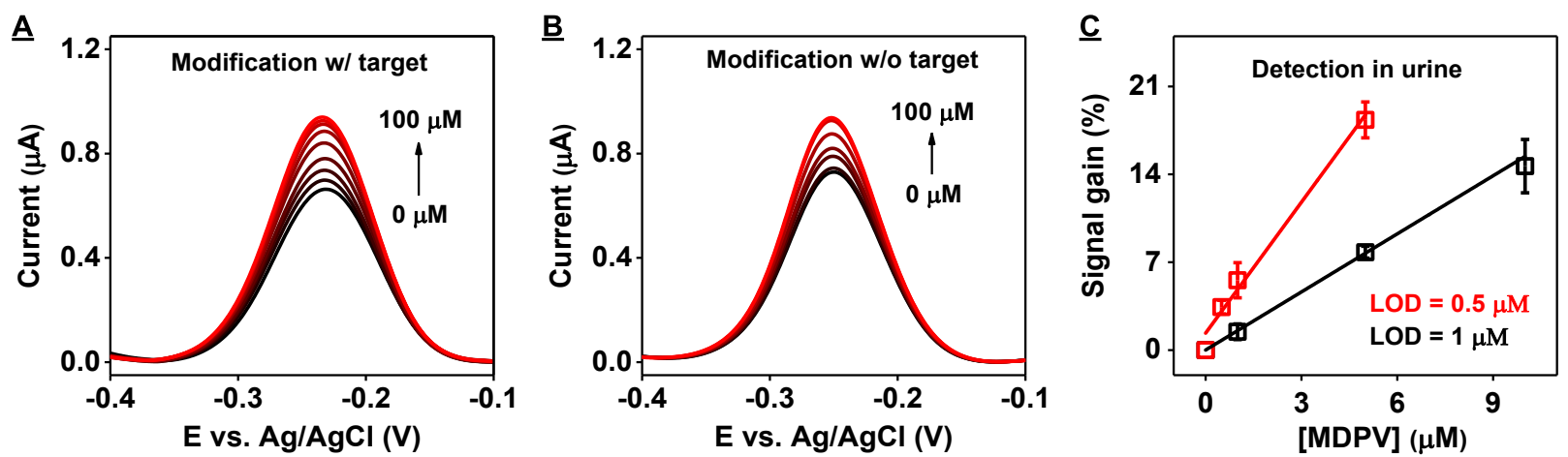

Figure S16. Detection of MDPV in 50\% urine using electrodes modified with SC-34-MB via (A) target-assisted immobilization in low-salt Tris buffer with $50 \mu \mathrm{M}$ MDPV or (B) conventional means in high-salt PBS. (C) Calibration curves derived from spectra shown in $\mathbf{A}$ (red) and $\mathbf{B}$ (black). Error bars represent the standard deviation of measurements from three independently fabricated electrodes.
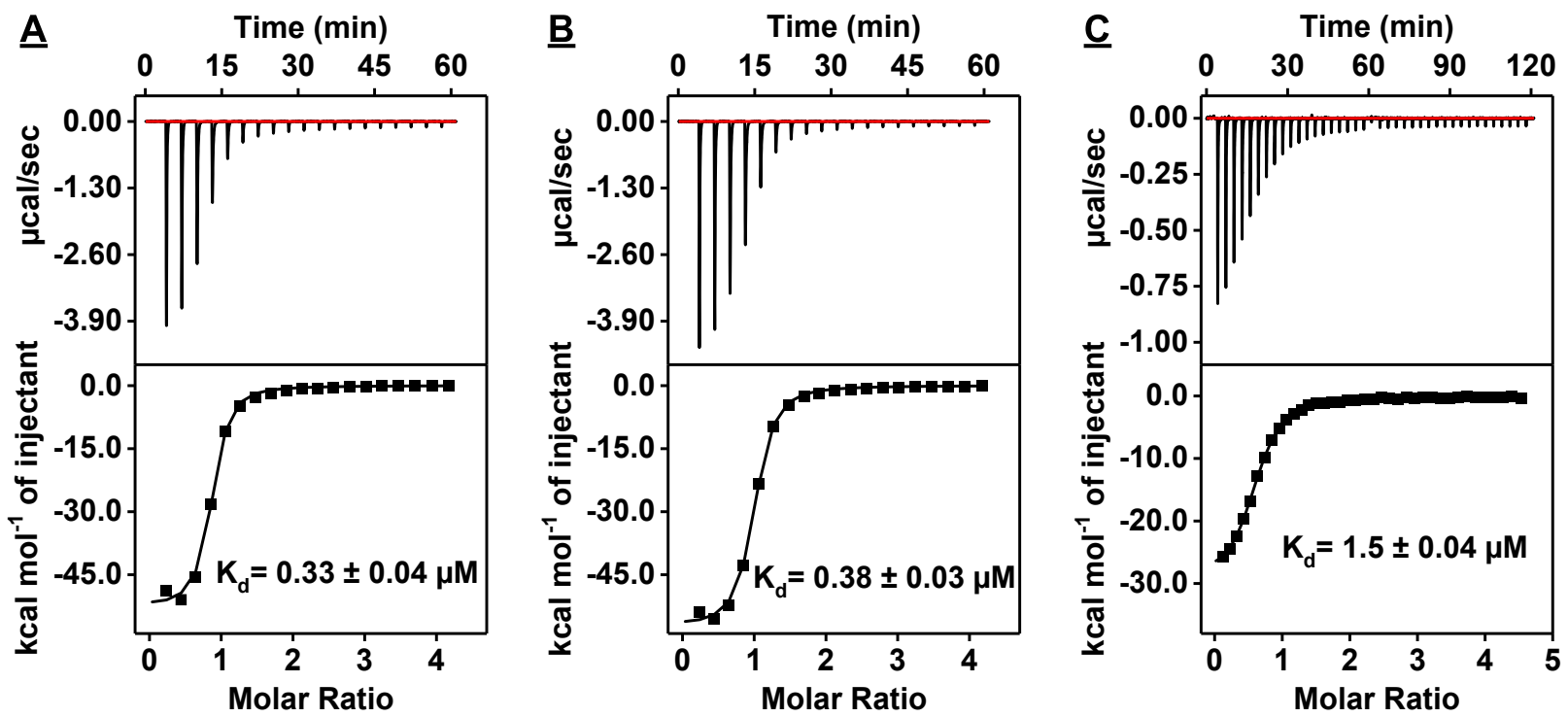

Figure S17. Characterization of the affinity of SC-34 for MDPV using ITC. Top panels present raw data showing the heat generated from each titration of MDPV to SC-34 in (A) low-salt Tris buffer, (B) low-salt PBS, or (C) high-salt PBS, while bottom panels show the integrated heat of each titration after correcting for dilution heat of the titrant. ITC data were fitted with a single-site binding model. 


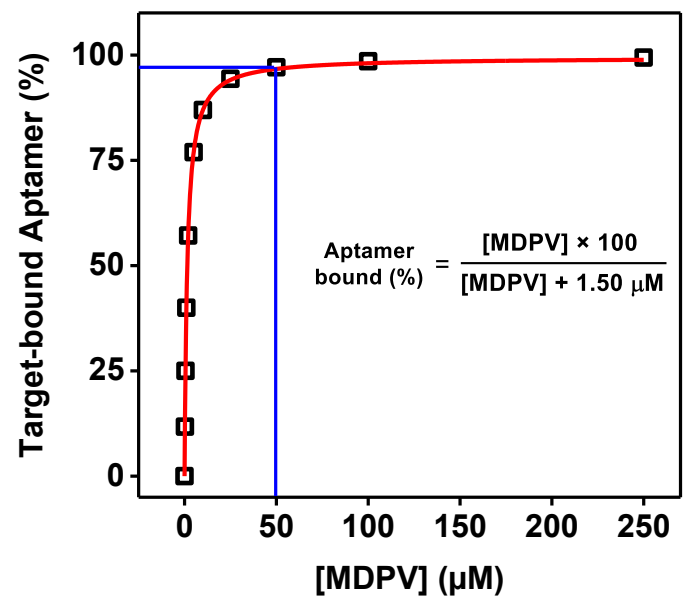

Figure S18. Simulated binding curve for SC-34 based on affinity for MDPV in high-salt PBS (KD $=1.50 \mu \mathrm{M})$. The blue lines indicate that $97 \%$ of the aptamer is bound in the presence of $50 \mu \mathrm{M}$ MDPV.
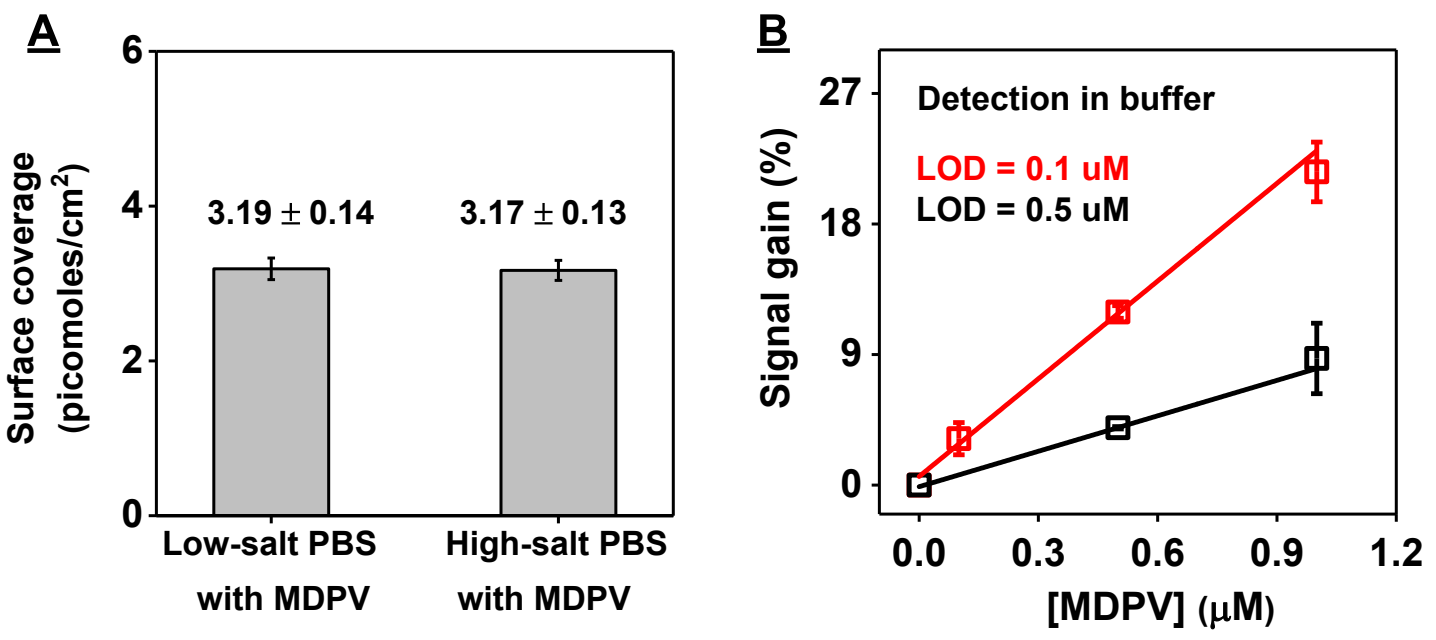

Figure S19. Performance of electrodes fabricated with SC-34-MB via target-assisted immobilization in different buffers. (A) Surface coverage of electrodes fabricated by immobilizing the aptamer in low-salt or high-salt PBS with $50 \mu \mathrm{M}$ MDPV. (B) Calibration curve and LOD for MDPV detection using electrodes modified with SC-34-MB via target-assisted immobilization in low-salt (red) or high-salt PBS (black). Error bars represent the standard deviation of measurements from three independently fabricated electrodes. 

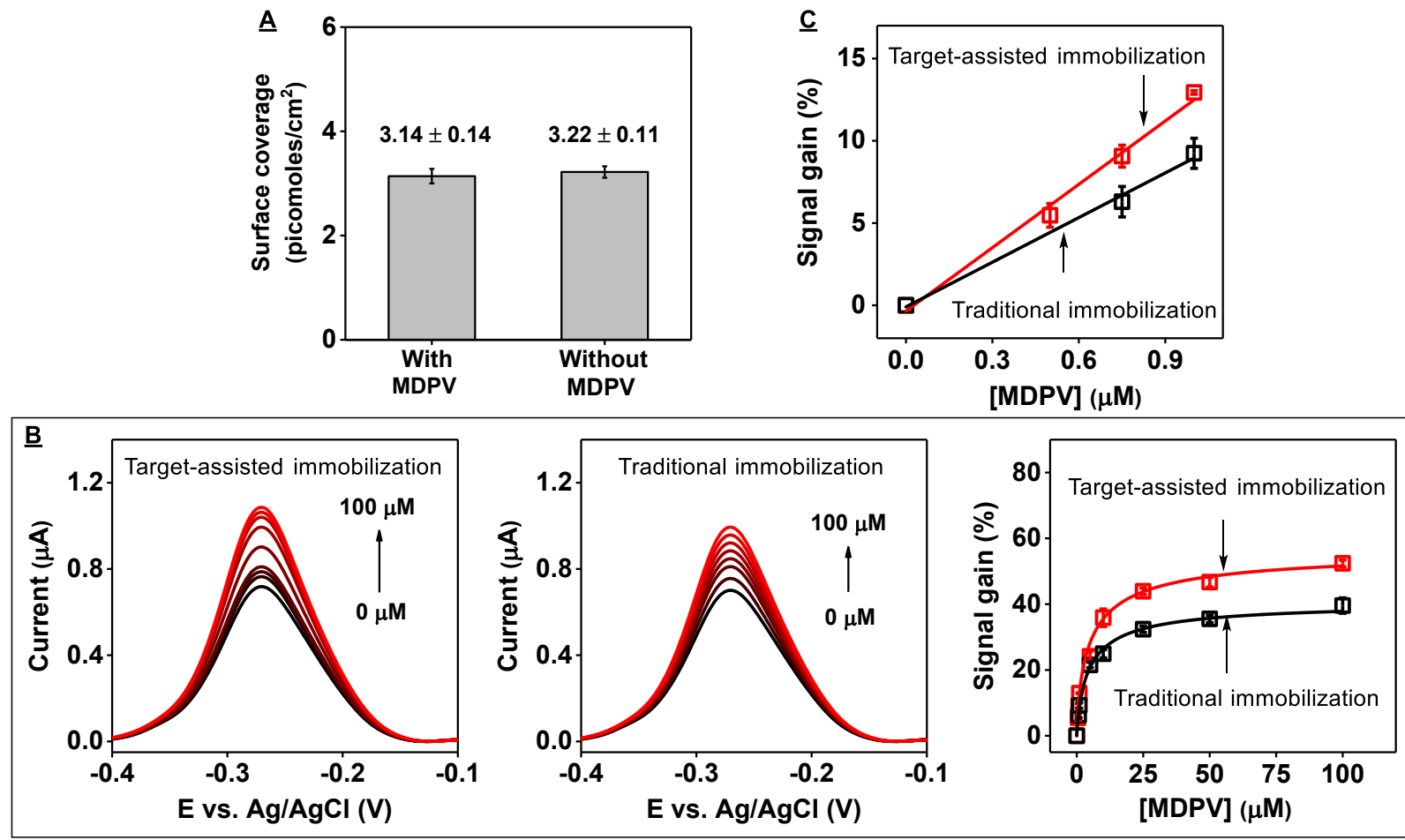

Figure S20. Performance of electrodes fabricated with SC-34-MB in the presence or absence of $50 \mu \mathrm{M}$ MDPV in high-salt Tris buffer. (A) Aptamer surface coverage of fabricated electrodes. (B) SWV measurements from electrodes fabricated via target-assisted aptamer immobilization approach (left) or traditional aptamer immobilization (middle) in the presence of $0-1000 \mu \mathrm{M}$ MDPV. The derived calibration curve is shown at right. (C) Linear ranges from the curves shown in B. Error bars represent the standard deviation of measurements from three independently fabricated electrodes. 


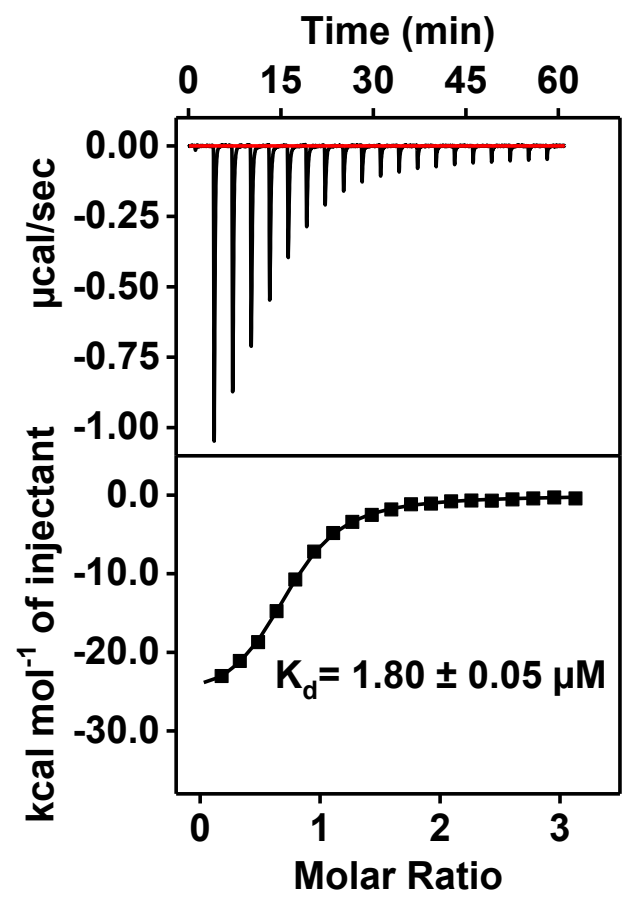

Figure S21. ITC characterization of SC-34 affinity for MDPV in high-salt Tris buffer. Top panels present raw data, showing the heat generated from each titration of MDPV to SC-34, while bottom panels show the integrated heat of each titration after correcting for dilution heat of the titrant. ITC data were fitted with a single-site binding model.

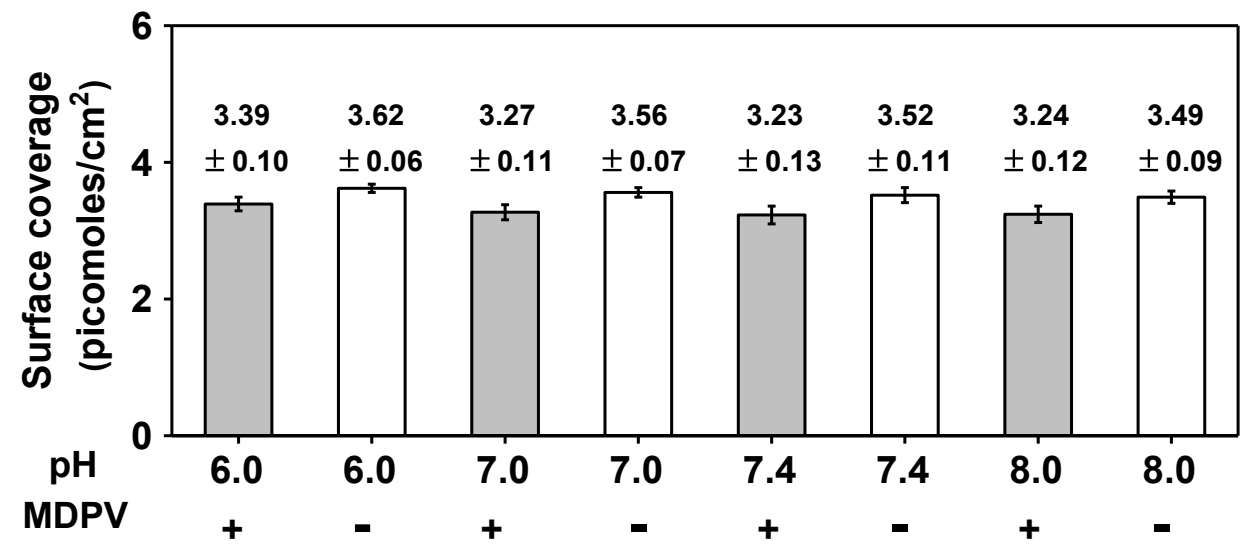

Figure S22. Aptamer surface coverages of electrodes fabricated with SC-34-MB in the absence (white bars) or presence of $50 \mu \mathrm{M}$ MDPV (gray bars) in low-salt PBS with a $\mathrm{pH}$ of 6.0, 7.0, 7.4 or 8.0. Error bars represent the standard deviation of measurements from three independently fabricated electrodes. 

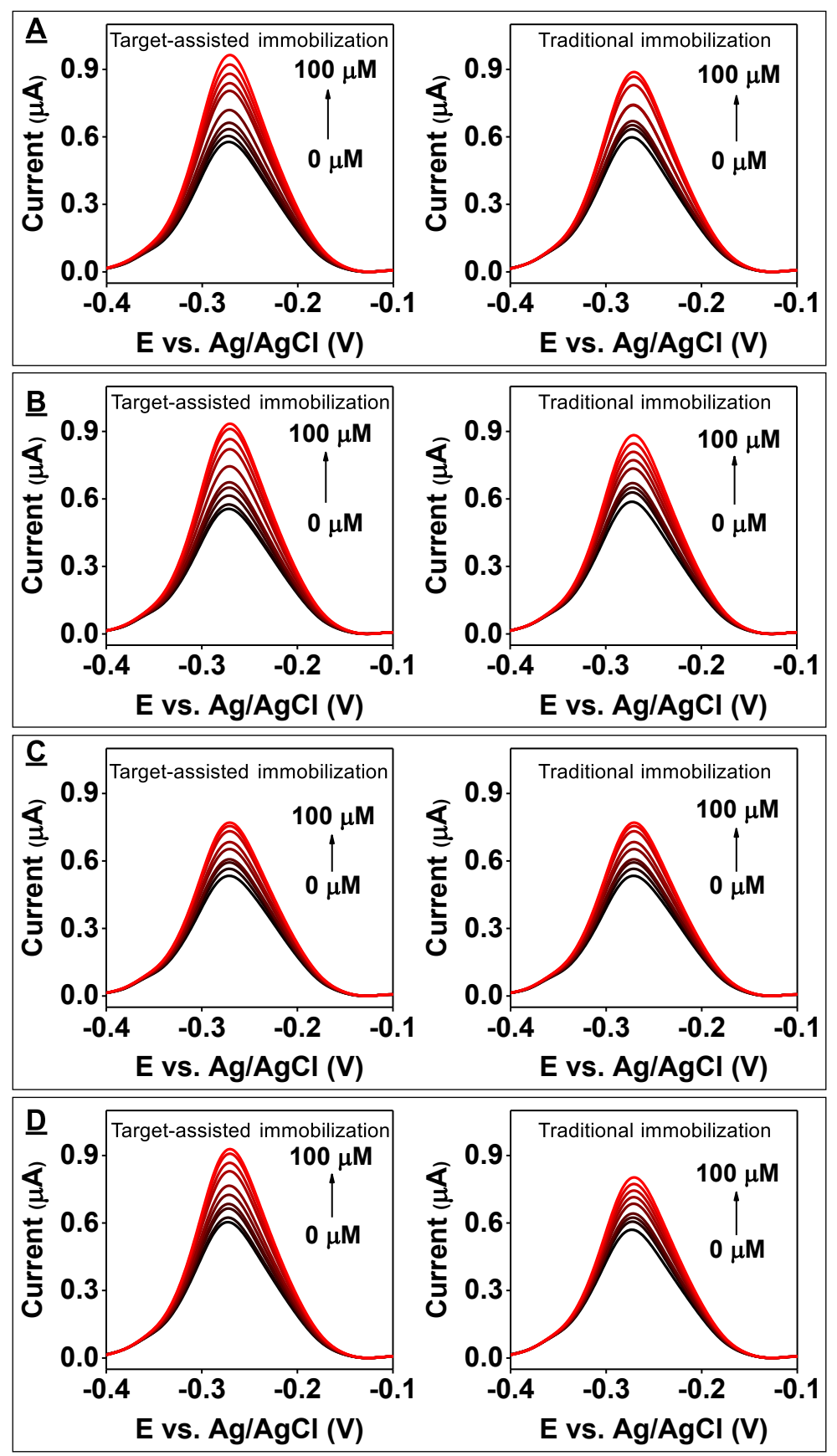

Figure S23. E-AB sensor performance using electrodes modified with SC-34-MB in the presence (target-assisted immobilization) or absence (traditional immobilization) of $50 \mu \mathrm{M}$ MDPV in lowsalt PBS at pH (A) 8.0, (B) 7.4, (C) 7.0 and (D) 6.0. SWV spectra for electrodes prepared under these two immobilization conditions and then exposed to 0-100 $\mu \mathrm{M}$ MDPV. Error bars represent the standard deviation of measurements from three independently fabricated electrodes. 

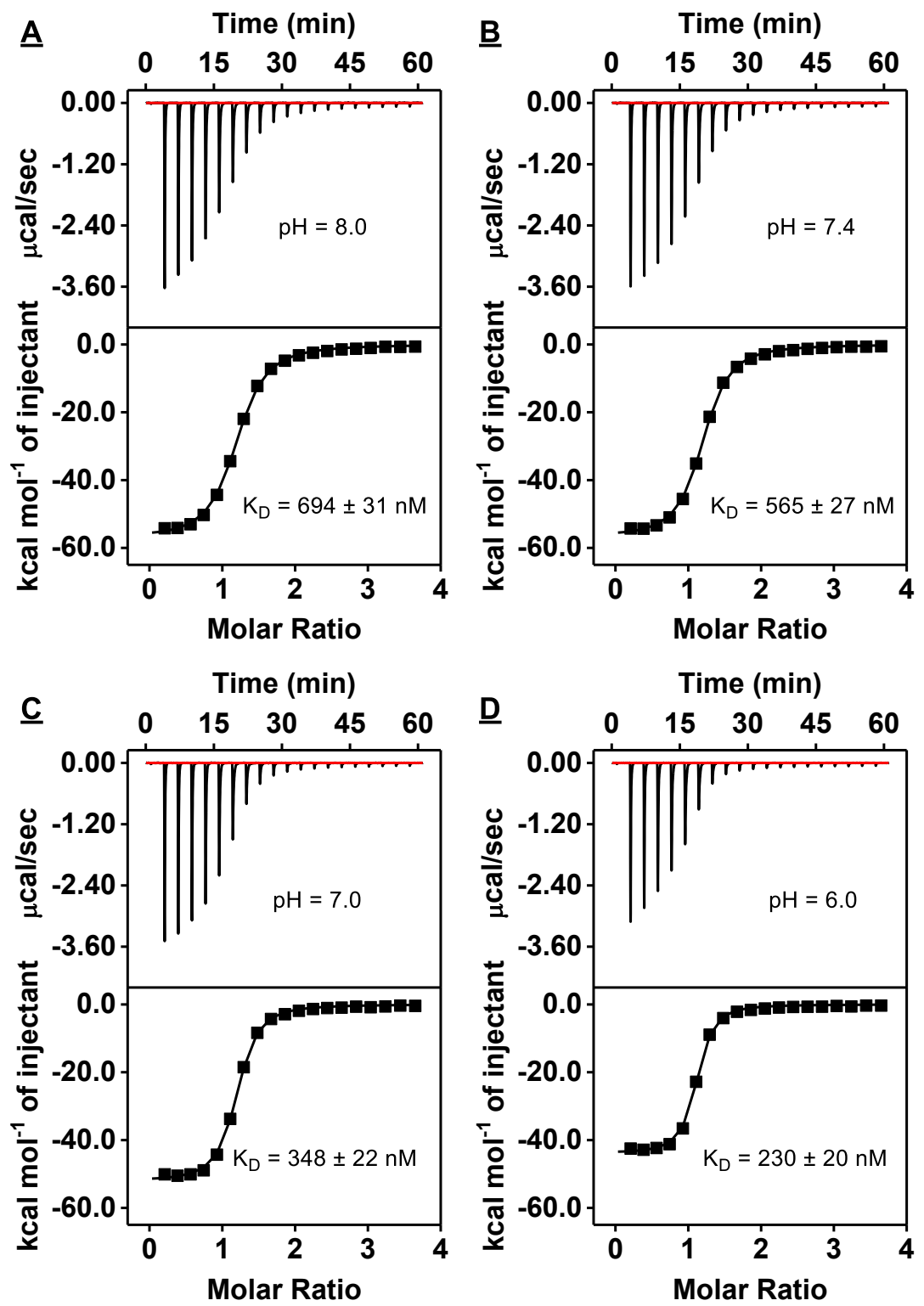

Figure S24. Characterization of the $\mathrm{pH}$-dependence of SC-34 affinity for MDPV using ITC. Top panels present raw data showing the heat generated from each titration of MDPV to SC-34 in lowsalt PBS at pH (A) 8.0, (B) 7.4, (C) 7.0, or (D) 6.0, while bottom panels show the integrated heat of each titration after correcting for dilution heat of the titrant. ITC data were fitted with a singlesite binding model. 